\title{
SAÚDE DO TRABALHADOR NA ATENÇÃO BÁSICA: ANÁLISE A PARTIR DE UMA EXPERIÊNCIA MUNICIPAL
}

\author{
THE WORKERS' HEALTH IN PRIMARY CARE: AN ANALYSIS OF A MUNICIPAL EXPERIENCE
}

\author{
Maria Dionísia do Amaral Dias ${ }^{1}$ \\ Grazielle Cristina dos Santos Bertolini ${ }^{2}$ \\ Aparecida Linhares Pimenta ${ }^{3}$
}

Resumo Este texto apresenta um relato da experiência de implantação de ações de saúde do trabalhador nos serviços de atenção básica no município de Amparo, no estado de São Paulo, ocorrida durante a gestão do período 2001 a 2008, visando contribuir na premente necessidade do SUS de implantação das ações de saúde do trabalhador. A análise realizada pelas autoras, protagonistas na gestão do processo, ressalta alguns dos elementos-chave para a efetiva implantação de ações de saúde do trabalhador na rede de atenção básica. Dentre eles, a Estratégia Saúde da Família (ESF), ao lado de outros elementos - como o modelo de matriciamento com ação pedagógica contínua e participação nos colegiados de gestão, a educação permanente e o protagonismo dos trabalhadores da saúde -, mostrouse um facilitador do processo. Como elementos estruturantes da construção efetivada, apontam-se o modelo de gestão colegiada adotado pela secretaria e a forma de implantação do Centro de Referência de Saúde do Trabalhador no município. Todo esse arranjo promoveu uma interlocução permanente entre as equipes de saúde, da atenção básica e da referência especializada que foi indispensável para a experiência ocorrida.

Palavras-chave saúde do trabalhador; educação permanente em saúde; saúde da família; atenção primária à saúde.
Abstract This article presents an account of the experience of implementing worker's health actions in primary care services in the municipality of Amparo, state of São Paulo, which occurred during the 2001 to 2008 administration period, aiming to contribute to SUS' urgent need to implement of workers' health actions. The analysis made by the authors, actors in managing the process, highlights a few of the key elements for the effective implementation of workers' care actions in the primary care network. Among them, the Family Health Strategy (FHS), along with other elements - such as the mixed-base model with continuous pedagogical action and participation in collegiate management, continuing education, and the role of the health workers - proved to be a facilitator process. Structural elements in the construction that was made include the collegiate management model adopted by the department and the way the Occupational Health Reference Center was deployed in the municipality. This arrangement has fostered permanent dialogue among the health, primary care, and referral specialist teams that has been indispensable to the experience.

Keywords occupational health; continuing education in health; family health; primary health care. 


\section{Introdução}

Os textos legais do Sistema Único de Saúde (SUS) incorporam a concepção da saúde do trabalhador (ST) como questão de saúde coletiva, o que implica o desenvolvimento de um conjunto de ações de promoção, prevenção e controle de risco como responsabilidades do setor saúde, a serem realizadas em conjunto com as ações assistenciais.

A atenção em ST é recente no sistema público de saúde brasileiro e mesmo com a implantação do SUS ainda não houve extensão de cobertura capaz de garantir acesso à maioria dos trabalhadores acometidos por agravos relacionados ao trabalho.

As primeiras ações de saúde pública voltadas para a saúde do trabalhador organizaram-se a partir da década de 1980, com equipes e serviços especializados em estreita integração com o movimento sindical (Mendes e Dias, 1991; Lacaz, 1997 e 2007; Maeno e Carmo, 2005), porém muitas vezes funcionando como espaços isolados na rede de saúde. Isso dificultava o esforço de construção da integralidade, que pressupõe a integração dos serviços do sistema de saúde, com a atenção primária operando como base real do sistema e devendo ser resolutiva em $80 \%$ das situações de saúde.

Atualmente, o desenvolvimento das ações de ST se dá de forma muito diversificada nos estados e municípios, tanto em relação à quantidade de Centros de Referência de Saúde do Trabalhador (Cerests) quanto em relação à qualidade das ações de ST, configurando-se como um atraso no cumprimento da lei, o que se reflete na morbi-mortalidade dos trabalhadores (Brasil, 2004).

Um dos desafios da ST é conseguir que o conjunto de trabalhadores e gestores do SUS incorpore na sua prática cotidiana a compreensão de que o trabalho é um dos determinantes do processo saúde-doença e de que é necessário o envolvimento de todo o sistema de saúde para garantir o cuidado integral aos trabalhadores. A atenção básica particularmente, como porta de entrada desse sistema, deve estar preparada para oferecer atenção adequada aos trabalhadores. Segundo a Política Nacional de Atenção Básica (Pnab),

(...) a atenção básica caracteriza-se por um conjunto de ações de saúde, no âmbito individual e coletivo, que abrangem a promoção e a proteção da saúde, a prevenção de agravos, o diagnóstico, o tratamento, a reabilitação e a manutenção da saúde. É desenvolvida por meio do exercício de práticas gerenciais e sanitárias democráticas e participativas, sob forma de trabalho em equipe, dirigidas a populações de territórios bem delimitados, pelas quais assume a responsabilidade sanitária, considerando a dinamicidade existente no território em que vivem essas populações. Utiliza tecnologias de elevada complexidade e baixa densidade, que devem resolver os problemas de saúde de maior frequência e relevância em seu território. É o contato preferencial dos usuários com os sistemas de saúde. Orien- 
ta-se pelos princípios da universalidade, da acessibilidade e da coordenação do cuidado, do vínculo e continuidade, da integralidade, da responsabilização, da humanização, da equidade e da participação social (Brasil, 2007, p. 12).

Para cumprir o seu papel, as equipes de atenção básica necessitam do apoio permanente de equipes especializadas que atuem não somente como serviço de referência que recebe o doente e o trata isoladamente, mas que operem de forma articulada, partilhando o cuidado ao usuário e dando subsídios para a ampliação do repertório de cuidados das equipes de atenção básica. Para garantir a integralidade da atenção e a ampliação do acesso para os trabalhadores, as ações de saúde do trabalhador não podem ser desenvolvidas exclusivamente como prerrogativa de serviços especializados; precisam ser incorporadas não só pelas equipes de atenção básica, como também pelos serviços de vigilância, garantindo-se ações de prevenção e controle de riscos nos ambientes de trabalho, conforme previsto em diversas normas e recomendações do SUS. 4

Apesar dos vinte anos de história do SUS, ainda existe certa distância entre o que está normatizado e o que ocorre no cotidiano dos diferentes serviços, pois o processo de construção de um sistema que tem como princípios a universalização do acesso e a integralidade da atenção enfrenta resistências, e sua construção vem ocorrendo na contramão do modelo político hegemônico e dos interesses do capital.

O campo da ST faz parte desse processo, mas com particularidades que tornam sua implantação ainda mais complexa, tais como: o contexto social da tensão capital-trabalho; os entraves políticos no âmbito municipal, principalmente em relação às ações de vigilância, o que faz com que muitos gestores não invistam nesse campo; o desconhecimento de muitos gestores do SUS quanto à concepção e ações de saúde do trabalhador; o despreparo de alguns profissionais de saúde para desenvolver ações nessa área, tanto em relação à capacidade técnica quanto ao imaginário (pois muitos acreditam que as questões de saúde relacionadas ao trabalho são mais uma questão jurídica do que de saúde ou temem desdobramentos futuros que lhes tragam algum transtorno, tais como emissão de relatórios técnicos e outros documentos); dentre outros aspectos. Daí a ST ser, talvez, uma das áreas de maior defasagem em relação à implantação no SUS, apesar de estar entre as prioridades do Pacto pela Vida para 2008/2009 e 2010/2011.

Trabalhadores que adoecem, ficam mutilados ou morrem em decorrência de suas atividades profissionais são atendidos nas emergências, nos hospitais e nas unidades básicas de saúde (UBS), como usuários do SUS. É preciso que a rede dos sistemas municipais de saúde se organize para desenvolver ações mais efetivas, a fim de prevenir agravos e sequelas e assistir de forma mais qualificada aqueles com doenças e acidentes de trabalho. 
Com o objetivo de contribuir para esse debate e compartilhar uma experiência considerada bem-sucedida, este texto relata e analisa a implantação de ações de ST na rede de atenção básica do município de Amparo, no estado de São Paulo, no período de 2003 a 2008. Ressalta-se que as autoras são protagonistas do processo relatado, integralmente implicadas com a experiência, mas a analisam já com certo distanciamento, em razão da desvinculação de duas das autoras daquela rede.

\section{Ações integradas e cuidado integral}

A experiência que aqui se analisa trata da implantação das ações de ST no município de Amparo, que ocorreu na gestão 2001 a 2008, com foco principal na atenção básica. ${ }^{5}$

A gestão municipal de saúde, entendendo ser a ST uma das responsabilidades do SUS e que ela exige o envolvimento, de maneira articulada, de todo o sistema de saúde, iniciou a implantação do programa no município em 2003. O processo de implantação do programa de ST no município de Amparo ocorreu de forma gradativa e englobou quatro conjuntos de ações: a assistência ao trabalhador, iniciada na atenção básica e, posteriormente, apoiada pelo Centro de Referência de Saúde do Trabalhador (Cerest); a vigilância em saúde do trabalhador, desenvolvida primeiramente pela Vigilância em Saúde e, depois, em conjunto com o Cerest; a educação em saúde, visando levar à população e aos trabalhadores as informações e o debate da relação saúde-trabalho; e a capacitação dos profissionais da rede municipal de saúde, visando primeiramente ao início das ações nas Unidades de Saúde da Família (USFs) e na Vigilância em Saúde, usando a educação permanente como ferramenta para qualificar a atenção aos trabalhadores, e fortalecendo a prevenção sob a ótica da vigilância à saúde.

A articulação das ações de ST na rede municipal de saúde envolveu diversas estratégias: apresentação das diretrizes do programa ao Colegiado de Saúde da Família; ${ }^{6}$ discussão do programa e incorporação das ações, com agendamento prévio, na atenção básica com cada equipe das USFs; 7 capacitação em serviço da equipe de Vigilância em Saúde (sanitária e epidemiológica), para o desenvolvimento de ações de vigilância em saúde nos ambientes de trabalho. Desde o início, a aceitação das equipes quanto à proposta já indicava a viabilidade do programa.

A Estratégia Saúde da Família (ESF) foi adotada pelo município para organizar toda a atenção básica, com objetivo de concretizar o acesso igualitário e integral dos usuários. O município contava, em 2008, com 20 equipes do Programa Saúde da Família (PSF), distribuídas em 14 unidades, com cobertura de $90 \%$ da população. 
A implantação da assistência em ST no município de Amparo iniciouse nas USFs, valorizando-se o seu potencial na atenção e no cuidado às pessoas, que, segundo a Pnab, se caracteriza por

(...) considerar o sujeito em sua singularidade, complexidade, integralidade e inserção sociocultural, buscando a promoção de sua saúde, a prevenção e o tratamento de doenças e a redução de danos ou de sofrimentos que possam comprometer suas possibilidades de viver de modo saudável (Brasil, 2007, p. 12).

Na sensibilização com as equipes de saúde da família, discutiu-se a determinação do trabalho em muitos processos de adoecimento, a importância de se considerar esse aspecto na vida da maioria das pessoas atendidas pelas equipes e, também, como as mesmas encaravam o fato de lidar com mais uma problemática do núcleo familiar. Além disso, foram discutidas as principais atividades econômicas e ocupações do território de abrangência da USF.

A ESF propicia o envolvimento da equipe com o seu território e com a clientela adscrita, de maneira a responsabilizar-se por todos os problemas da sua região, entre os quais se inclui o trabalho. Nessa etapa, foi constatado o quanto aquelas equipes conheciam seu território e percebiam em seu cotidiano muitas questões de saúde do trabalhador. Isso fez a maioria dos profissionais receber as discussões sobre o tema como uma oportunidade de apoio em situações em que se sentiam despreparados e solitários para encontrar condutas adequadas.

Após essa primeira etapa de reuniões com as equipes, foram realizadas capacitações formais para os profissionais da atenção básica que abrangeram todas as USFs, e que também contaram com a participação de alguns profissionais do Ambulatório de Especialidades e da futura equipe do Cerest: curso de atualização para médicos e enfermeiras, com o tema "LER/Dort: diagnóstico e tratamento", incluindo uma "Introdução à saúde do trabalhador" (16 horas); e curso de "Introdução à saúde do trabalhador para técnicos de enfermagem e agentes comunitários de saúde" (8 horas).

Outra atividade importante no processo de implantação da ST no município foi o I Encontro de Saúde do Trabalhador, realizado em maio de 2003 e organizado pela Secretaria Municipal de Saúde de Amparo (SMSA) e por sindicatos de trabalhadores (dois deles com abrangências diversas) da região - metalúrgicos, químicos e indústria de alimentos -, visando à articulação com outras instituições no intuito de levar a discussão para a sociedade em geral e para os profissionais de saúde em particular. O evento, realizado em dois dias, contou com a participação de cerca de 150 pessoas, entre profissionais de saúde da SMSA, dirigentes sindicais, trabalhadores de categorias diversas, profissionais de recursos humanos, saúde e segurança de empresas e alunos do curso técnico de segurança no trabalho. A programação abordou 
aspectos diversos da questão da saúde dos trabalhadores e da atuação do SUS nessa área, propiciando aos participantes um panorama geral da situação. $\mathrm{O}$ encontro tornou-se atividade anual do Cerest, passando a ser realizado próximo ao aniversário de inauguração do serviço, sempre levando à comunidade um tema relevante, apresentado por profissionais de destaque nacional na área.

Esse processo criou as condições para que a implantação e o funcionamento do Cerest Amparo fossem bastante diferentes da maioria dos Cerests implantados, no mesmo período, em diversos municípios do país. Ele foi inaugurado em 2004, mais de um ano após o início da implantação do programa, já habilitado para integrar a Rede Nacional de Atenção Integral à Saúde do Trabalhador (Renast) do SUS.

O Cerest atende trabalhadores com suspeita de doenças relacionadas ao trabalho e funciona como serviço especializado de referência, sendo que a porta de entrada para os trabalhadores são as equipes das USFs. O encaminhamento do trabalhador se dá quando se esgotam as possibilidades de se estabelecer nexo causal entre os problemas de saúde e as situações de trabalho e de assistência ao trabalhador pelos profissionais da atenção básica.

No período analisado, o Cerest também desenvolveu ações de vigilância em ambientes de trabalho de forma integrada, com a Vigilância Sanitária e com a Vigilância Epidemiológica, quanto às informações referentes à saúde do trabalhador. O serviço era responsável, ainda, por articular ações de saúde do trabalhador no SUS municipal e na microrregião com outros órgãos públicos - Delegacia Regional do Trabalho, agência local do Instituto Nacional do Seguro Social (INSS), Procuradoria do Trabalho - e outras instituições - sindicatos de trabalhadores e representações patronais -, ampliando as ações de promoção da saúde dos trabalhadores.

A equipe do Cerest estava composta por enfermeira, médico, psicóloga, terapeuta ocupacional, técnicos em enfermagem, engenheiro de segurança do trabalho, técnicos de segurança do trabalho, agentes administrativos e agentes de limpeza.

Para concretizar as ações de ST na rede básica, a equipe do Cerest adotou o modelo de matriciamento das equipes, estruturado em um conjunto de ações permanentes: a) participação nos colegiados das equipes das USFs e no Colegiado de Coordenadores; b) ação pedagógica, que consistia na disponibilidade permanente para discussão e orientação aos profissionais com base em casos concretos atendidos na atenção básica; c) articulação entre referência e contrarreferência, com retorno direto à equipe, por escrito ou por telefone, dos casos encaminhados, especificando-se a conduta adotada, com suas devidas justificativas; d) educação permanente em saúde, incluindo cursos para capacitação técnica; e e) desenvolvimento de atividades conjuntas na comunidade. 
A postura de parceria assumida pela equipe da ST desde o início das atividades foi fundamental para que os profissionais das USFs se dispusessem a partilhar o cuidado com o trabalhador.

Entendemos o apoio matricial como um arranjo institucional criado para promover a interlocução entre as equipes de saúde, interlocução que, juntamente com a educação permanente, constituiu estratégia importante para a efetiva implantação da saúde do trabalhador na atenção básica de Amparo. O apoio matricial objetiva assegurar, de modo dinâmico e interativo, a retaguarda especializada para equipes e profissionais de referência, sugerindo modificações nas relações entre os níveis hierárquicos em sistemas de saúde; além da retaguarda assistencial, objetiva-se, com ele, produzir um espaço em que ocorra o intercâmbio sistemático de conhecimentos entre as várias especialidades e profissões, de modo a tornar horizontal a relação entre equipes de saúde (Campos e Domitti, 2007; Brasil, 2003).

Outras estratégias de ação foram importantes no cotidiano do trabalho, tais como:

- padronização de fluxos com as USFs, com a elaboração do "Passo a passo de saúde do trabalhador", contendo informações básicas, expostas de forma objetiva, distribuído aos serviços.

- publicação de decreto tornando obrigatória nos serviços de saúde da SMSA a notificação de todos os acidentes e doenças relacionados ao trabalho (decreto municipal no 2.834, de 3/8/2004).

- publicação de lei tornando obrigatória em todos os serviços de saúde do município a notificação de acidentes e doenças do trabalho (lei municipal no 3.028, de 13/9/2004).

- supervisão e orientação das equipes das USFs, com realização de reuniões periódicas.

- elaboração de material educativo em saúde do trabalhador, com edição da série "Fique atento", visando facilitar a comunicação de questões relevantes aos trabalhadores (material utilizado em todos os serviços de saúde).

- análise da morbidade ambulatorial dos fluxos de encaminhamento e por perfil de atividade econômica, com o objetivo de planejar ações específicas no território, em conjunto com equipe da USF. Dois exemplos dessas ações são: 1) a implementação de um grupo de apoio psicossocial para trabalhadores portadores de LER/Dort numa USF localizada em bairro que concentrava as maiores empresas do município e com o grande número de operários residentes; 2) identificando-se que a questão dos trabalhadores rurais, e da contaminação por agrotóxicos, não se manifestava de forma clara, sendo necessária, portanto, a orientação e a intervenção das USFs que abrangiam áreas rurais, o Cerest realizou projetos integrados com as unidades de saúde, procedendo ao levantamento das características da atividade rural e das famílias envolvidas na atividade, implementando a ação 
educativa direta, o mapeamento da exposição a produtos químicos e a avaliação de expostos. O projeto contou com o apoio de diversas instituições parceiras, principalmente da área agrícola, e do Conselho Municipal de Desenvolvimento Rural.

- as atividades de educação permanente em saúde faziam parte das atribuições do Cerest e constavam anualmente do seu plano de trabalho e da programação anual da SMS Amparo, sendo parte integrante do modelo de matriciamento, como já mencionado. As capacitações, planejadas de acordo com a demanda da rede e com base na análise dos dados epidemiológicos, eram dirigidas à totalidade das equipes, sendo oferecidas sempre em horário de trabalho. A educação permanente em saúde era tida pela SMSA como atividade estratégica da gestão, dentro da diretriz de valorização dos profissionais, o que facilitava a programação específica da área de ST.

- realização de dois grandes eventos educativos por ano, considerando a regularidade como estratégia de manter o tema ST na pauta social: Semana de Saúde do Trabalhador, 8 dirigida aos profissionais de saúde e segurança, com atividades diversificadas voltadas à mobilização social, à informação aos trabalhadores e à reflexão de tema atual; e o Encontro de Saúde do Trabalhador, conforme já comentado anteriormente.

As estratégias acima, bem como os vários dispositivos criados, possibilitaram a efetiva incorporação das ações de ST na rede municipal, num processo que incluía planejamento, execução das ações, avaliação e muita comunicação institucional envolvendo as equipes do Cerest e das USFs, as quais de fato, em parceria com os especialistas, cuidavam dos trabalhadores.

\section{Análise e considerações finais}

A análise da experiência desenvolvida no município de Amparo de 2001 a 2008 permite que sejam apontados alguns elementos-chave para a efetiva implantação de ações de ST na rede de atenção básica.

A ESF, pela sua abrangência - cobertura de $90 \%$ da população amparense - e pelo processo de produção do cuidado pelas equipes, mostrou-se um facilitador do processo de implantação da ST. Contudo, é preciso ressaltar que Cerest e ESF não são suficientes para que ocorra uma integração entre esses níveis, seja de maneira espontânea, seja por medidas normativas. Assim, a integração foi o resultado de muito trabalho, enfrentamento de resistências e conflitos, e construção compartilhada do cuidado ao trabalhador, com base na valorização dos diferentes saberes e sujeitos envolvidos na atenção à saúde.

É preciso considerar ainda que a integralidade do cuidado depende da integração do sistema, e essa só é possível quando se estabelecem relações de 
parcerias e de corresponsabilidades entre as equipes de saúde, relações que resultam, principalmente, do envolvimento dos trabalhadores de saúde e da compreensão de que nenhum profissional ou equipe dominam sozinhos todos os conhecimentos necessários para a produção de cuidado integral.

Não é possível a incorporação da saúde do trabalhador na atenção básica sem levar em consideração os trabalhadores de saúde e sua atividade profissional, sendo esse um aspecto essencial da gestão em saúde (Merhy e Feuerwerker, 2009) e do campo da ST. O protagonismo dos trabalhadores da saúde na experiência de Amparo foi decisivo; ele permitiu que a definição de quais as melhores ações estratégicas para a implantação/implementação de ações de ST na atenção básica fosse sendo construída no processo.

Nesse sentido, a educação permanente e o modelo de matriciamento adotado pela equipe do Cerest, com ação pedagógica contínua e participação nos colegiados de gestão, aparecem como outros elementos-chave que facilitaram o compartilhamento de responsabilidades por todos os atores envolvidos no processo de cuidar da saúde dos trabalhadores.

Um dos elementos estruturantes dessa construção adotado pela secretaria foi a gestão colegiada, ou seja, a gestão partilhada por todos os atores envolvidos diretamente ou indiretamente no processo de gestão, planejamento, assistência, promoção e proteção à saúde.

Segundo diversos estudiosos de gestão e planejamento do SUS, a gestão colegiada é um dispositivo potente de democratização, propiciando a construção coletiva e objetivando a estruturação de uma assistência de qualidade. Denominamos colegiados os espaços destinados às reuniões periódicas de equipe ou de gestores, voltadas à discussão dos problemas e à organização do processo de produção do cuidado, da gestão ou da vigilância à saúde.

A estrutura organizacional da SMS Amparo contava com diversos espaços colegiados: o das equipes em cada serviço de saúde, o dos coordenadores de USFs, o dos coordenadores de serviços de referência e o da gestão central da secretaria. Os colegiados constituíam espaços democráticos para a análise de problemas, o enfrentamento de conflitos, a construção de consensos e a pactuação de objetivos e estratégias, tanto em relação às questões do sistema de saúde como um todo quanto em relação à micropolítica presente no cotidiano dos serviços. As pactuações obtidas com base na discussão colegiada são mais sustentáveis e duradouras, e produzem uma visão compartilhada por todos e enriquecida pela variedade de pontos de vista, competências e funções dos membros do colegiado.

Outro aspecto que favoreceu o desenvolvimento das ações de ST foi a forma de implantação do Cerest no município, pois a habilitação e a inauguração do serviço ocorreram após a rede municipal de saúde estar sensibilizada e já ter implementado algumas ações de saúde do trabalhador. Porém, isso implicou a capacitação concomitante não apenas da equipe do Cerest, mas 
também da rede básica de saúde, o que trouxe diversas dificuldades para o processo. Apesar da realização de ações de assistência em ST pelas equipes da rede básica de saúde de Amparo, com particular adesão dos médicos generalistas, havia necessidade de se investir na ampliação do cuidado, com a incorporação pelas USFs, em seu cotidiano, da lógica das ações coletivas e da vigilância à saúde do trabalhador, acionando mais o Cerest e a Vigilância Sanitária para intervenções nos locais de trabalho.

A experiência de Amparo demonstra que é viável a execução das ações de ST em municípios de pequeno e médio porte, mediante a incorporação de ações na atenção básica. No entanto, a ST tem de ser assumida como política pública da área da saúde, com cofinanciamento tripartite compatível com seus custos. É preciso que os gestores e os trabalhadores do SUS assumam suas responsabilidades em relação às violências cometidas com os trabalhadores nos seus locais de trabalho, superando-se a situação atual de atendimento de trabalhadores já doentes e mutilados por acidentes de trabalho. O conjunto de ações dirigidas à ST deve ter como objetivo a defesa da vida dos trabalhadores, que resulta em menores gastos sociais e econômicos, com reflexos diretos também no sistema de saúde.

\section{Notas}

1 Professora do Departamento de Saúde Pública da Faculdade de Medicina de Botucatu, Universidade Estadual Paulista (Unesp-Botucatu), Botucatu, São Paulo, Brasil. Doutora em Psicologia Social pela Pontifícia Universidade Católica de São Paulo (PUC-SP). <dionisia@fmb.unesp.br>

Correspondência: Departamento de Saúde Pública, Faculdade de Medicina de Botucatu, Unesp, Caixa Postal 549, Botucatu, São Paulo, CEP 18618-970.

2 Professora da Faculdade de Enfermagem da Universidade São Francisco, Bragança Paulista, São Paulo, Brasil, e enfermeira da Secretaria Municipal de Saúde de Amparo, Amparo, São Paulo, Brasil. Especialista em Saúde do Trabalhador pela Faculdade de Saúde Pública, Universidade São Paulo (FSP/USP). <grazielle@amparo.sp.gov.br>

3 Secretária municipal de Saúde de Diadema, Diadema, São Paulo, Brasil. Doutora em Saúde Coletiva pela Universidade Estadual de Campinas (Unicamp). <aparecida.pimenta@diadema.sp.gov.br>

4 Por exemplo, a portaria/MS GM n ${ }^{\circ} 1.125 / 2005$, estabelece "que as ações em saúde do trabalhador desenvolvidas pelo SUS sejam organizadas em todos seus níveis de atenção" (art. $2^{\circ}$ ).

5 Apesar de o Cerest de Amparo ser um serviço de referência regional na Rede Nacional de Atenção Integral à Saúde do Trabalhador (Renast), o presente texto irá enfocar a 
experiência no município sede, onde havia governabilidade da gestão municipal de saúde. Destaca-se que todas as atividades de educação permanente foram ofertadas aos municípios da área de abrangência (16 no total), havendo participação frequente apenas de parte deles.

6 O Colegiado de Saúde da Família reunia os coordenadores de todas as USF, a secretária municipal de saúde e o grupo de coordenação da Atenção Básica.

7 Em todas as USFs, as equipes reuniam-se semanalmente em horário de trabalho, o que facilitava a inclusão de temas a serem discutidos com as equipes.

8 Realizada próximo a datas importantes para a ST: 28 de abril - Dia Mundial em Memória das Vítimas de Acidentes e Doenças do Trabalho; $1^{\circ}$ de Maio - Dia do Trabalho; 2 de maio - Dia Nacional de Luta contra o Assédio Moral.

\section{Referências}

AMPARO. Secretaria Municipal de Saúde de Amparo (SMS-Amparo). Plano municipal de saúde 2005-2008. Amparo: SMS Amparo, 2005.

Relatório de gestão. Amparo: SMS Amparo, 2004.

Relatório de gestão. Amparo: SMS Amparo, 2005.

Relatório de gestão. Amparo: SMS Amparo, 2006.

Relatório de gestão. Amparo: SMS Amparo, 2007.

Relatório de gestão. Amparo: SMS Amparo, 2008.

BRASIL. Ministério da Saúde. Secretaria de Atenção à Saúde. Coordenação de Saúde Mental; Coordenação de Gestão da Atenção Básica. Saúde mental e atenção básica. O vínculo e o diálogo necessários. Inclusão das ações de saúde mental na atenção básica. Brasília: Editora do Ministério da Saúde, 2003. (Circular conjunta $\mathrm{n}^{\circ} 1 / 3$ )

Departamento de Ações Programáticas Estratégicas. Área Técnica de Saúde do(a) Trabalhador(a). Politica nacional de saúde do trabalhador. Brasília: Ministério da Saúde, 2004. Mimeografado.

Departamento de

Atenção Básica. Política nacional de atenção básica. 4. ed. Brasília: Ministério da Saúde, 2007. (Série E, Legislação de Saúde, Pactos pela Saúde, 4).

CAMPOS, Gastão Wagner de Sousa; DOMITTI, Ana Carla. Apoio matricial e equipe de referência: uma metodologia para gestão do trabalho interdisciplinar em saúde. Cadernos de Saúde Pública, Rio de Janeiro, v. 23, n. 2, p. 399-407, fev. 2007.

DIAS, Elizabeth Costa; HOEFEL, Maria da Graça. O desafio de implementar as ações de saúde do trabalhador no SUS: a estratégia da Renast. Ciência \& Saúde Coletiva, Rio de Janeiro, v. 10, n. 4, p. 817-828, 2005.

LACAZ, Francisco Antonio de Castro. O campo saúde do trabalhador: resgatando conhecimentos e práticas sobre as relações trabalho-saúde. Cadernos de Saúde Pública, Rio de Janeiro, v. 23, n. 4, p. 757-766, 2007.

Saúde dos trabalhadores: cenário e desafios. Cadernos de Saúde Pública, Rio de Janeiro, v. 13, supl. 2, p. 7-19, 1997. 
MAENO, Maria; CARMO, José Carlos do. Saúde do trabalhador no SUS: aprender com o passado, trabalhar o presente, construir o futuro. São Paulo: Hucitec, 2005.

MENDES, René; DIAS, Elizabeth Costa. Da medicina do trabalho à saúde do trabalhador. Revista de Saúde Pública, São Paulo, v. 25, n. 5, p. 3-11, 1991.

MERHY, Emerson Elias; FEUERWERKER, Laura C. Macruz. Novo olhar sobre as tecnologias de saúde: uma necessidade contemporânea. In: MANDARINO, Ana Cristina de Souza; GOMBERG, Estélio (org.). Leituras de novas tecnologias e saúde. Aracaju: Editora da UFS, 2009.

PIMENTA, Aparecida L.; CAPISTRANO FILHO, David (Org.). Saúde do trabalhador. São Paulo: Hucitec, 1988.

Recebido em 05/04/2010

Aprovado em 17/05/2010 